\title{
Factorials of real negative and imaginary numbers - A new perspective
}

\author{
Ashwani K Thukral
}

\begin{abstract}
Presently, factorials of real negative numbers and imaginary numbers, except for zero and negative integers are interpolated using the Euler's gamma function. In the present paper, the concept of factorials has been generalised as applicable to real and imaginary numbers, and multifactorials. New functions based on Euler's factorial function have been proposed for the factorials of real negative and imaginary numbers. As per the present concept, the factorials of real negative numbers, are complex numbers. The factorials of real negative integers have their imaginary part equal to zero, thus are real numbers. Similarly, the factorials of imaginary numbers are complex numbers. The moduli of the complex factorials of real negative numbers, and imaginary numbers are equal to their respective real positive number factorials. Fractional factorials and multifactorials have been defined in a new perspective. The proposed concept has also been extended to Euler's gamma function for real negative numbers and imaginary numbers, and beta function.
\end{abstract}

Keywords: Factorials of negative numbers; Factorials of imaginary numbers; Pi function; Fractional factorials; Multifactorials; Gamma function; Beta function

\section{Background}

The factorial of a positive integer, $n$, is defined as,

$$
\begin{aligned}
& n !=1.2 .3 \ldots .(n-1)(n)=\prod_{k=1}^{n} k, \\
& 0 !=1
\end{aligned}
$$

The factorials of positive integers follow the recurrence relation,

$$
n !=n(n-1) !, n \geq 1
$$

The factorials of negative integers cannot be computed, since for $n=0$, the recurrence relation,

$$
(n-1) !=\frac{n !}{n}
$$

involves a division by zero. Research on the interpolation of factorials started with correspondence among Leonhard Euler, Daniel Bernoulli and Christian Goldbach in the year 1729 (Refer to the correspondence reproduced by Dartmouth College 2014; and Luschny 2014a). Bernoulli

Correspondence: akthukral.gndu@gmail.com

Department of Botanical \& Environmental Sciences, Guru Nanak Dev University, Amritsar 143005, India in the year 1729 gave an interpolating function of factorials as an infinite product (Gronau 2003). Euler in the year 1730 proved that the integral,

$$
x !=\Pi(x)=\int_{0}^{1}(-\ln t)^{x} d t=\int_{0}^{\infty} t^{x} e^{-t} d t, x>-1,
$$

gives the factorial of $x$ for all real positive numbers (Srinivasan 2007). Euler's factorial function, also known as the Pi function, $\Pi(x)$, follows the recurrence relation for all positive real numbers.

$$
\Pi(x+1)=(x+1) \Pi(x), x \geq 0
$$

In 1768 , Euler defined the gamma function, $\Gamma(\mathrm{z})$, and extended the concept of factorials to all real negative numbers, except zero and negative integers. $\Gamma(\mathrm{z})$, is an extension of the Pi function, with its argument shifted down by 1 . Also known as the Euler's integral of the second kind (Gautschi 2008), it is a convergent improper integral defined as follows:

$$
\Gamma(z)=\int_{0}^{\infty} t^{z-1} e^{-t} d t
$$

\section{包 Springer}

(c) 2014 Thukral; licensee Springer. This is an Open Access article distributed under the terms of the Creative Commons Attribution License (http://creativecommons.org/licenses/by/4.0), which permits unrestricted use, distribution, and reproduction in any medium, provided the original work is properly credited. 
The Euler's gamma function is related to the Pi function as follows:

$$
\Pi(x)=\Gamma(x+1)=x !
$$

The notation '!' for the factorial function was introduced by C. Kramp in the year 1808 (Wolfram Research 2014a,b). Legendre in 1808 gave the notation ' $\Gamma$ ' to the Euler's gamma function (Gronau 2003). Gauss introduced the notation

$$
\Pi(s)=\Gamma(s+1),
$$

which was subsequently abandoned and replaced with Legendre's notation (Weistein 2014b). C.F. Gauss made important contributions to derive several important properties of the gamma function (Srinivasan 2007). Anglani and Barlie (2007) gave the additive representation of factorials. The gamma function is extended to all complex numbers, with a real part $>0$, except for at zero and negative integers. Figure 1 gives the curve for gamma function (Eqn. 2). At negative integers, the gamma function has simple poles, making it a meromorphic function (Figure 1).

It may be seen that the gamma function defined for $x>-1$ has been used to interpolate the factorials on the real negative axis. Similarly, the factorials of complex numbers are calculated from the gamma function (Wolfram 2014b), such as,

$$
\begin{aligned}
& i !=\Gamma(1+i)=i \Gamma(i) \approx 0.498-0.155 i \\
& (-i) !=\Gamma(1-i)=-i \Gamma(-i) \approx 0.498+0.155 i \\
& (-1+i) !=\Gamma(i) \approx-1549-0.498 i
\end{aligned}
$$

and

$$
|\Gamma(i x)|=\sqrt{\frac{\pi}{x \sinh (\pi x)}},
$$

where $x$ is a real number. Among the other well defined functions for the factorials of real negative numbers are, Hadamard's gamma function (Davis 1959) and Luschny's factorial function (Luschny 2014b), both of which are continuous and positive at all real numbers. Roman (1992) defined the factorials of negative integers as under:

$$
\begin{array}{ll}
\llcorner n\urcorner !=n ! & \text { for } n \geq 0 \\
\llcorner n\urcorner !=\frac{(-1)^{-n-1}}{(-n-1) !} & \text { for } n<0
\end{array}
$$

where Roman $\lfloor n\rceil$ is defined as $n$, for $n \neq 0$, and 1 for $n=0$. Roman factorials of first few negative integers (Loeb 1995) are given in Table 1.

The other notable contributors to the field of factorials are J. Stirling, F.W. Newman, B. Riemann, H. Hankel, O. Holder, H. Bohr and J. Mollerup, and others (Wolfram Research 2014b). Dutka (1991) gave an account of the early history of the factorial function. Bhargava (2000) gave an expository account of the factorials, gave several new results and posed certain problems on factorials. Ibrahim (2013) defined the factorial of negative integer $n$ as the product of first $n$ negative integers. There are some other factorial like products and functions, such as, primordial, double factorial, multifactorials, superfactorial, hyperfactorials etc. (Wikipedia 2014a,b; Weistein 2014a).

It is seen that till now the definition of the factorials of real negative numbers is sought from the extrapolation of gamma and other functions. In the present paper, the

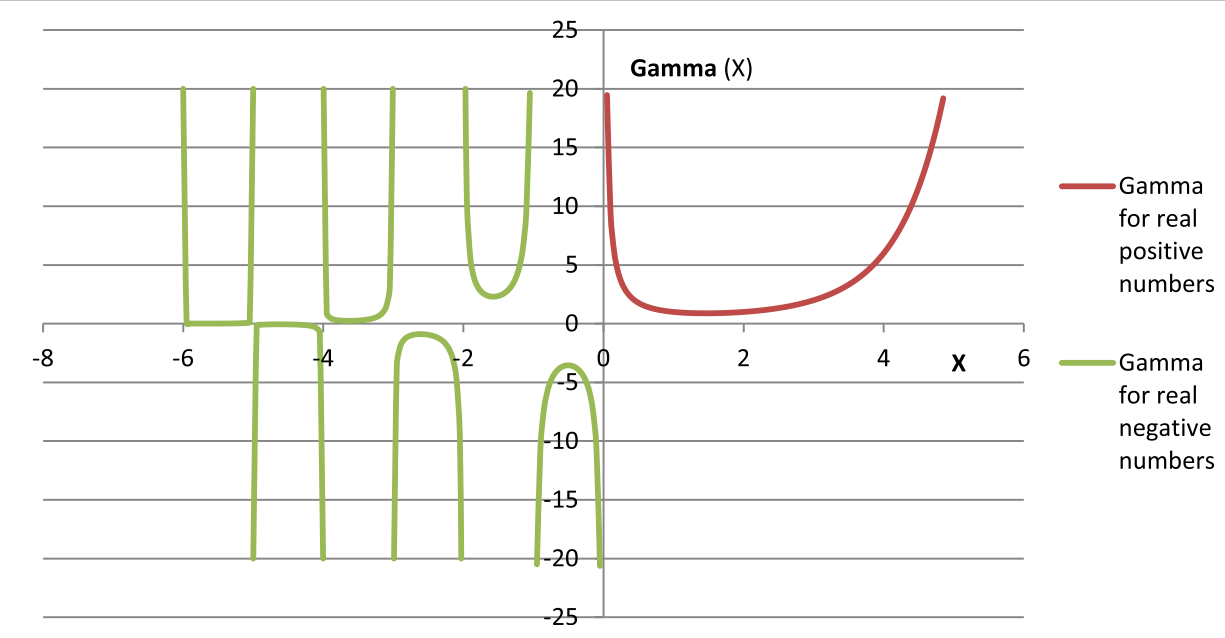

Figure $1 \mathrm{~A}$ plot of Euler's gamma function. 
Table 1 Roman factorials

\begin{tabular}{lc}
\hline $\boldsymbol{n}$ & Roman factorial $\mathbf{L} \boldsymbol{\mathbf { ~ } !}$ \\
\hline 0 & 1 \\
-1 & 1 \\
-2 & -1 \\
-3 & $1 / 2$ \\
-4 & $-1 / 6$ \\
-5 & $1 / 24$ \\
-6 & $-1 / 120$ \\
\hline
\end{tabular}

Eularian concept of factorials has been revisited, and new functions based on Euler's factorial function (Eqn. 1) have been defined for the factorials of real negative numbers and imaginary numbers.

\section{Factorials of real negative numbers}

Let $a_{\mathrm{n}}$ be a sequence of positive integers, $a_{\mathrm{n}=1,2,3, \ldots, n}$. Therefore,

$n !=1.2 .3 \ldots n$.

Multiplying each integer on the right hand side of $a_{\mathrm{n}}$ with a constant, $c \neq 0$, termed here as factorial constant, we get

$$
(c)^{n} n !=c(c 2)(c 3) \ldots(c n) .
$$

Putting $c=-1$ gives,

$$
(-1)^{n} n !=(-1)(-2)(-3) \ldots(-n) .
$$

The expression $(-1)^{n} n$ ! on the left hand side of Eqn. (4), gives the product of first $n$ consecutive negative integers and may be termed as the factorials of negative integers (Table 2). For convenience Eqn. (4) may be presented as $(-n)$ !.

In the present communication, a new function obtained from the Euler's factorial function (Eqn. 1) has been proposed to interpolate the factorials of real negative numbers as given below:

$$
(c z) !=\left(c^{z}\right) z !=\Pi(c, z)=c^{z} \int_{0}^{\infty} t^{z} e^{-t} d t, z>0
$$

where $z$ is a real positive number, and $c$ is a factorial constant not equal to zero, and $\Pi(c, z)$ is modified Euler's

Table 2 Factorials of some integers as per present concept

\begin{tabular}{cccc}
\hline $\boldsymbol{n}$ & $\boldsymbol{n !}$ & $-\boldsymbol{n}$ & $(-\boldsymbol{n}) !=\left[(-1)^{\boldsymbol{n}} \boldsymbol{n} !\right.$ \\
\hline 1 & 1 & -1 & -1 \\
2 & 2 & -2 & 2 \\
3 & 6 & -3 & -6 \\
4 & 24 & -4 & 24 \\
5 & 120 & -5 & -120 \\
\hline
\end{tabular}

factorial function. For $c=1$, the factorial for real positive numbers is defined as per Euler's factorial function (Eqn. 1). For $c=-1$, factorials of real negative numbers as described by Eqn. (5) can be interpolated as follows:

$$
\begin{aligned}
& (-z) !=(-1)^{z} z !=\Pi(-1, z)=(-1)^{z} \int_{0}^{\infty} t^{z} e^{-t} d t, z>0 \\
& \Pi(-1, z)=\int_{0}^{\infty}(-t)^{z} e^{-t} d t, z>0
\end{aligned}
$$$$
\text { Or, }
$$

where $\Pi(-1, z)$ is the factorial of the negative real number $(-z)$ as per the present concept. For the real negative axis, Eqn. (6) may be written as

$$
\Pi(-1, z)=\int_{-\infty}^{0} t^{z} e^{t} d t, z>0
$$

Analogous to factorials of real positive numbers, the factorials of real negative numbers, $\Pi(-1, \mathrm{z})$ may be given by the notation $(-z)$ !. Figure 2 gives the curves for the integral functions of factorials of real negative integers, $(-1),(-2),(-3)$, on the real negative axis. The area between a curve and the $\mathrm{X}$-axis gives the factorial of that number.

The factorials of some real negative numbers as given by Eq. (6) are given in Table 3. The factorial of a real negative number is a complex number, represented as

$$
\Pi(-1, z)=(-z) !=x+i y
$$

where $x$ is the real part and $y$ is imaginary. The factorial of 0 is 1 . At real negative integers the imaginary part is zero and the real part has alternating - and + signs, with the factorial of $(-1)$ being $(-1)$. The most important property that justifies the present concept is that the moduli of the complex factorials of real negative numbers are equal to the factorials of real positive numbers.

It is also seen from Table 3, that for real negative numbers, at half fractions the real part is zero, at $1 / 4$ fractions, the real and imaginary parts are equal, and at $3 / 4$ fractions the real and imaginary parts are equal in magnitude but opposite in +/- sign.

Factorials of real negative numbers as proposed above follow recurrence relations:

$$
\Pi(-1, z+1)=-(z+1) \Pi(-1, z) .
$$




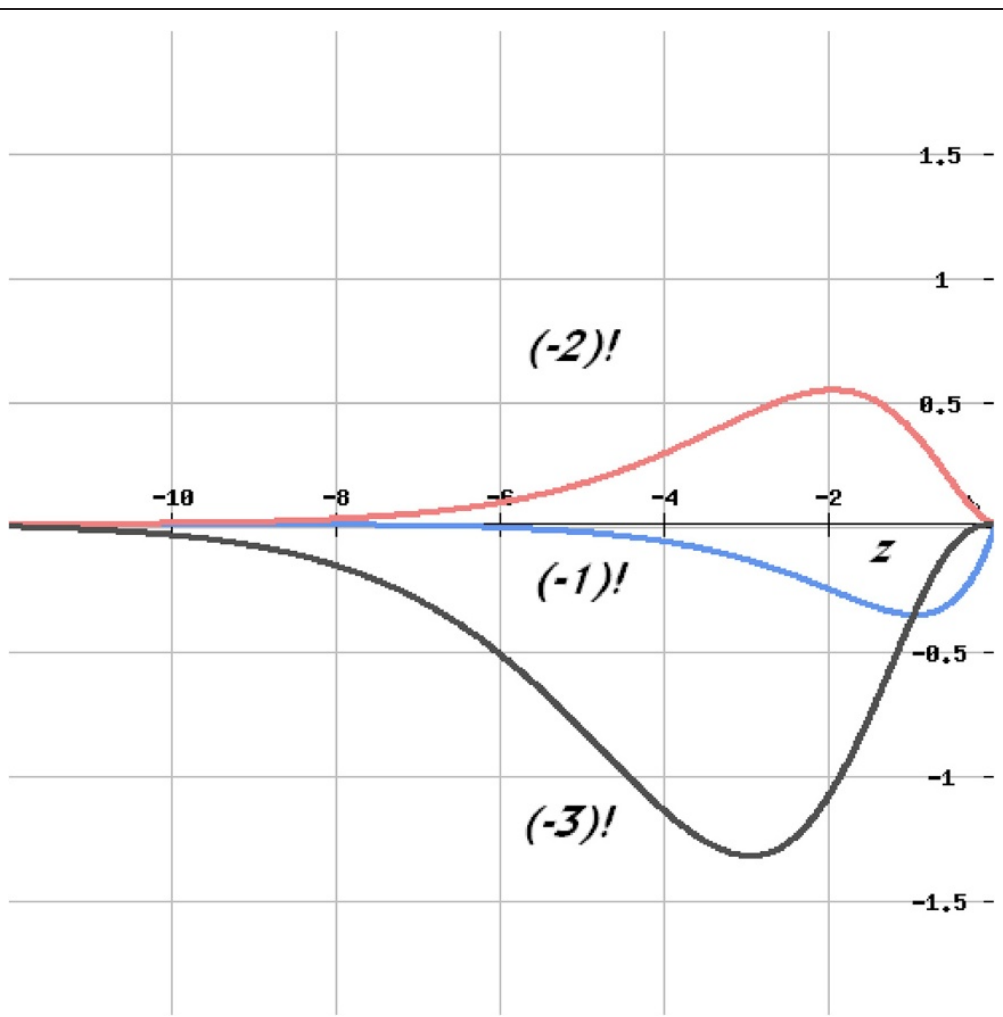

Figure 2 Curves for the integral functions of factorials of some negative integers on the real negative axis.

1.1 Factorials of half fractions of real negative numbers Let $\mathrm{Z}=n+0.5, n \geq 0$, then

$$
\begin{aligned}
& \Pi(-1, z)=(-1)^{z} \Pi(z)=(-1)^{0.5}(-1)^{n} \Pi(z) \\
& =i(-1)^{n} \Pi(z)
\end{aligned}
$$

Table 3 Complex factorials of some real negative numbers

\begin{tabular}{ccccc}
\hline & Real & Imaginary & Modulus & Im/Re \\
\hline$z$ & & \multicolumn{2}{c}{ Complex factorial of $(-z)$} & \\
0 & 1 & 0 & 1 & 0 \\
0.25 & 0.640 & $0.640 i$ & 0.906 & 1 \\
0.5 & 0 & $0.886 i$ & 0.886 & Comp Inf \\
0.75 & -0.649 & $0.649 i$ & 0.919 & -1 \\
1 & -1 & 0 & 1 & 0 \\
1.25 & -0.801 & $-0.801 i$ & 1.133 & 1 \\
1.5 & 0 & $-1.329 i$ & 1.329 & Comp Inf \\
1.75 & 1.137 & $-1.137 i$ & 1.608 & -1 \\
2 & 2 & 0 & 2 & 0 \\
2.25 & 1.802 & $1.802 i$ & 2.549 & 1 \\
2.5 & 0 & $3.323 i$ & 3.323 & Comp Inf \\
2.75 & -3.127 & $3.127 i$ & 4.422 & -1 \\
3 & -6 & 0 & 6 & 0 \\
\hline
\end{tabular}

Thus, the real part of the complex factorials of negative real numbers will be zero at negative half integers. At $z=-0.5$

$$
\Pi(-1,0.5)=\frac{\sqrt{\pi}}{2} i
$$

The factorials of -0.25 and -0.75 will be

$$
\begin{aligned}
& \Pi(-1,0.25)=(-1)^{0.25} \Pi(0.25) \approx 0.640+0.640 i \\
& \Pi(-1,0.75)=(-1)^{0.75} \Pi(0.75) \approx-0.640+0.640 i
\end{aligned}
$$

Figure 3 shows the Euler's factorials $\Pi(z)$ of real positive numbers (Eqn. 1) and factorials of real negative numbers as per proposed $\Pi(-1, z)$ function (Eqn. 6). Figure 4 gives the polar line graph between real and imaginary parts of complex factorials of negative real numbers, and Figure 5 describes the ratio of imaginary to real parts of complex factorials of real negative numbers. The polar graph of real $\mathrm{X}$-axis $v s$. imaginary $\mathrm{Y}$-axis for $\Pi(-1, z)$ function, and $\tan \theta,\left(\tan \frac{y}{x}\right)$ are shown in Figure 5 .

\subsection{Exponential function}

Exponential function can be represented in terms of factorials (Oldham et al. 2009): 


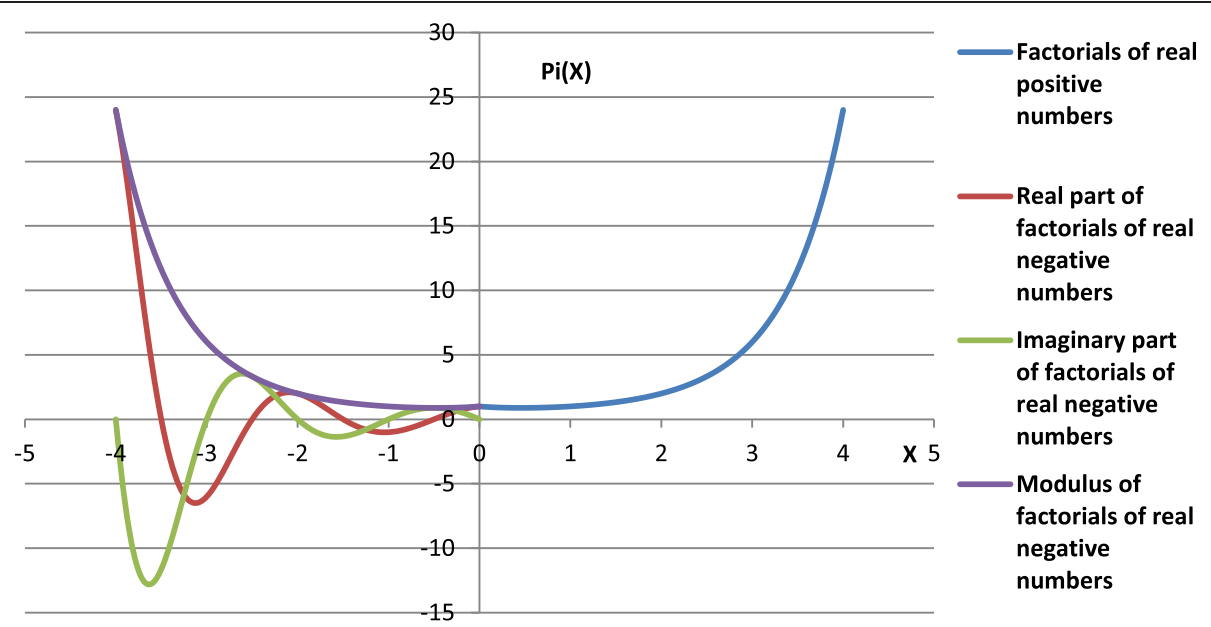

Figure 3 Factorials of real numbers using Euler's PI function (right) and the present $(-1, z)$ function (left).

$$
\begin{aligned}
\operatorname{Exp}(1) & =\frac{1}{0 !}+\frac{1}{1 !}+\frac{1}{2 !}+\frac{1}{3 !}+\ldots \\
& =\sum_{n=0}^{\infty} \frac{1}{n !}
\end{aligned}
$$

Substituting the positive factorials with negative factorials we get,

$$
\begin{aligned}
& \sum_{n=0}^{\infty} \frac{1}{(-n) !}=\frac{1}{0 !}+\frac{1}{(-1) !}+\frac{1}{(-2) !}+\frac{1}{(-3) !}+\ldots \\
& \quad=\frac{1}{(-1)^{0} 0 !}+\frac{1}{(-1)^{1} 1 !}+\frac{1}{(-1)^{2} 2 !}+\frac{1}{(-1)^{3} 3 !}+\ldots \\
& \quad=\frac{1}{0 !}-\frac{1}{1 !}+\frac{1}{2 !}-\frac{1}{3 !}+\ldots \\
& \quad=\operatorname{Exp}(-1)
\end{aligned}
$$

\section{Factorials of imaginary numbers}

Similar to the factorials of real positive and real negative integers as defined in Eqn. (3), we may define the factorials of imaginary positive integers as

$$
(i)^{n} n !=i(i 2)(i 3) \ldots(i n)
$$

For convenience $(i)^{n} n$ ! may be written as $(i n)$ ! In the integral form, Eqn. (8) may be written as,

$$
\begin{aligned}
& (i z) !=i^{z} z !=\Pi(i, z)=i^{z} \int_{0}^{\infty} t^{z} e^{-t} d t, z>0 \\
& \Pi(i, z)=\int_{0}^{\infty}(i t)^{z} e^{-t} d t, z>0
\end{aligned}
$$

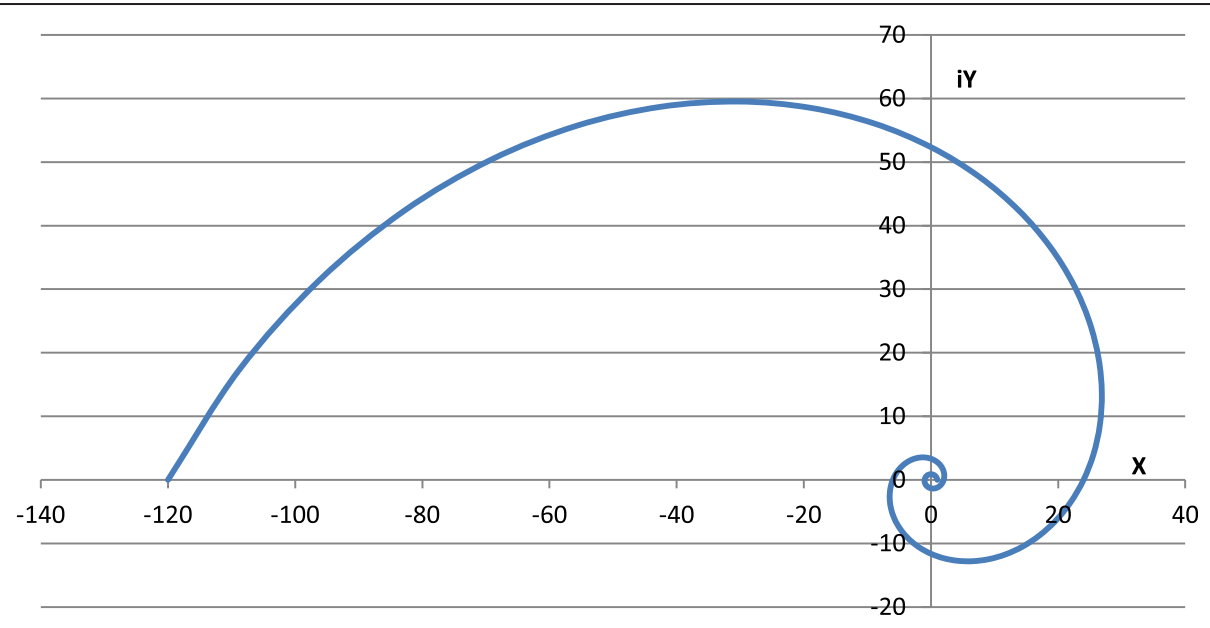

Figure 4 Polar line graph between real (X-axis) and imaginary (Y-axis) parts of factorials of negative real numbers using $(-1, z)$. 


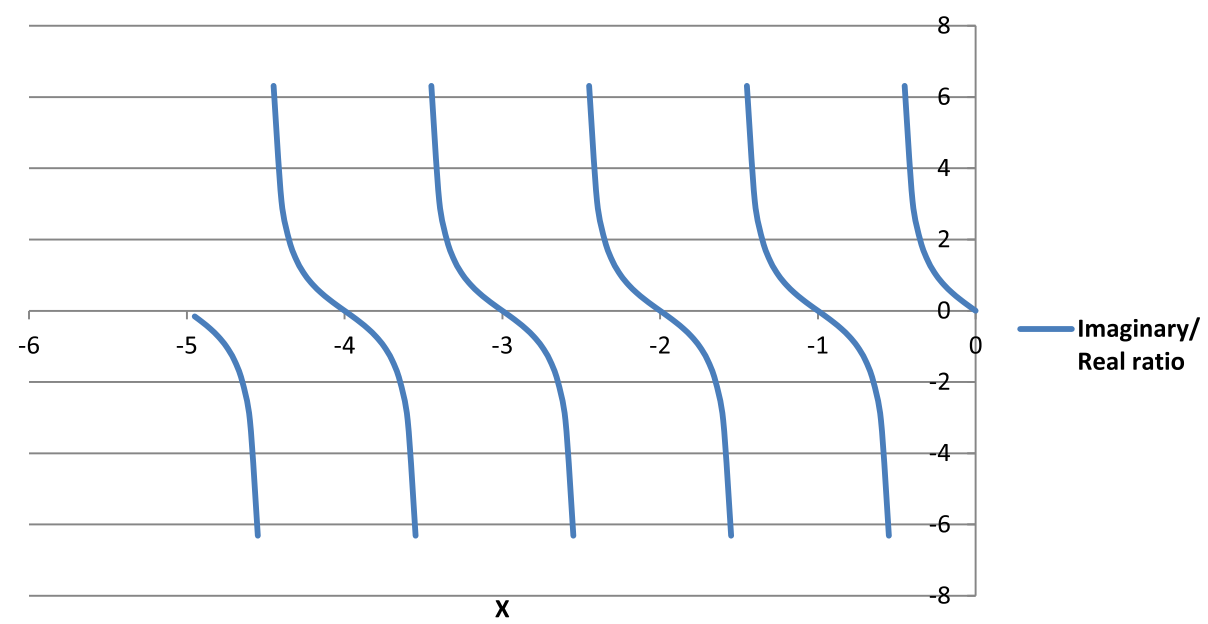

Figure 5 Ratio of imaginary to real part of factorials of negative numbers (Y-axis) using $(-1, z)$ function.

Similarly, factorials of imaginary negative integers may be defined as

$$
(-i)^{n} n !=(-i n) !=(-i)(-i 2)(-i 3) \ldots(-i n)
$$

The integral form of Eqn. (10) may be defined as

$$
\begin{aligned}
& (-i z) !=(-i)^{z} z !=\Pi(-i, z)=(-i)^{z} \int_{0}^{\infty} t^{z} e^{-t} d t, z>0 \\
& \Pi(-i, z)=\int_{0}^{\infty}(-i t)^{z} e^{-t} d t, z>0
\end{aligned}
$$

Complex factorials of imaginary numbers will be related to the factorials of the respective real numbers as follows:

$$
\begin{aligned}
& \Pi(i, z)=i^{z} \Pi(z) \\
& \Pi(-i, z)=(-i)^{z} \Pi(z)
\end{aligned}
$$

Complex factorials of some imaginary numbers as calculated from Eqn. $(12,13)$ are given in Table 4, and Figures 6, 7 and 8. The modulus of the complex factorial of an imaginary number $(i z)$ or $(-i z)$ is equal to the factorial of the respective real number $(z)$.

The factorial of an imaginary number $(i z)$ ! or $(-i z)$ ! may be represented as a product of the coefficient $\left(i^{z}\right)$ or $(-i)^{z}$ and $z$ ! (Eq. 12, 13). The coefficients of factorials of imaginary integers follow a periodicity of four (Table 5).

\begin{tabular}{|c|c|c|c|c|}
\hline \multirow[t]{2}{*}{$z$} & \multicolumn{4}{|c|}{ Complex factorial of (iz) } \\
\hline & Real & Imaginary & Modulus & $\operatorname{Im} / \operatorname{Re}$ \\
\hline 0 & 1 & 0 & 1 & 0 \\
\hline 0.25 & 0.837 & $0.346 i$ & 0.906 & 2.414 \\
\hline 0.5 & 0.626 & $0.626 i$ & 0.886 & 1 \\
\hline 0.75 & 0.351 & $0.849 i$ & 0.919 & 2.414 \\
\hline 1 & 0 & $i$ & 1 & Comp Inf \\
\hline 1.25 & -0.433 & $1.046 i$ & 1.133 & -0.414 \\
\hline 1.5 & -0.939 & $0.939 i$ & 1.329 & -1 \\
\hline 1.75 & -1.485 & $0.615 i$ & 1.608 & -2.414 \\
\hline 2 & -2 & 0 & 2 & 0 \\
\hline 2.25 & -2.355 & $-0.975 i$ & 2.549 & 2.414 \\
\hline 2.5 & -2.349 & $-2.349 i$ & 3.323 & 1 \\
\hline 2.75 & -1.692 & $-4.086 i$ & 4.422 & 0.414 \\
\hline 3 & 0 & $-6 i$ & 6 & Comp Inf \\
\hline$z$ & \multicolumn{4}{|c|}{ Complex factorial of $(-i z)$} \\
\hline 0 & 1 & 0 & 1 & 0 \\
\hline 0.25 & 0.837 & $-0.346 i$ & 0.906 & -0.414 \\
\hline 0.5 & 0.626 & $-0.626 i$ & 0.886 & -1 \\
\hline 0.75 & 0.351 & $-0.849 i$ & 0.919 & -2.414 \\
\hline 1 & 0 & $-i$ & 1 & Comp Inf \\
\hline 1.25 & -0.433 & $-1.046 i$ & 1.133 & 2.414 \\
\hline 1.5 & -0.939 & $-0.939 i$ & 1.329 & 1 \\
\hline 1.75 & -1.485 & $-0.615 i$ & 1.608 & 0.414 \\
\hline 2 & -2 & 0 & 2 & 0 \\
\hline 2.25 & -2.355 & $0.975 i$ & 2.549 & -2.414 \\
\hline 2.5 & -2.349 & $2.349 i$ & 3.323 & -1 \\
\hline 2.75 & -1.692 & $4.086 i$ & 4.422 & -0.414 \\
\hline 3 & 0 & $6 i$ & 6 & Comp Inf \\
\hline
\end{tabular}

Factorials of imaginary numbers follow recurrence relations:

$$
\begin{aligned}
& \Pi(i, z+1)=i(z+1) \Pi(i, z) \\
& \Pi(-i, z+1)=-i(z+1) \Pi(-i, z)
\end{aligned}
$$

Table 4 Complex factorials of some imaginary numbers 


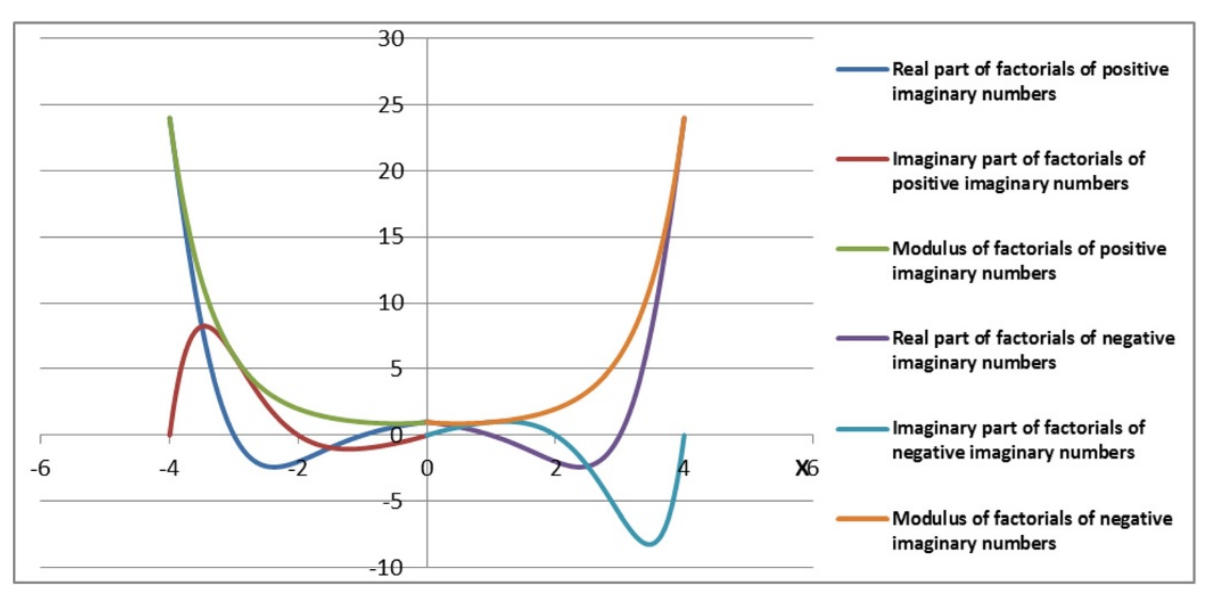

Figure 6 Graph of complex factorials of imaginary numbers.

2.1 Factorials of half fractions of imaginary numbers Let $z=n+0.5, n \geq 0$, then

$$
\begin{aligned}
& \Pi(i, z)=i^{z} \Pi(z)=i^{0.5} i^{n} \Pi(z), \\
& =\frac{i+1}{\sqrt{2}} i^{n} \Pi(z) \approx(0.7170+0.7170 i) i^{n} \Pi(z)
\end{aligned}
$$

Similarly, for imaginary negative numbers,

$$
\begin{aligned}
& \Pi(-i, z)=(-i)^{z} \Pi(z)=\left(-i^{0.5}\right)(-i)^{n} \Pi(z) \\
& =\frac{i-1}{\sqrt{2}}(-i)^{n} \Pi(z) \approx(0.7071-0.7071 i)(-i)^{n} \Pi(z)
\end{aligned}
$$

Factorials of $0.5 i$ and $-0.5 i$ are given as

$$
\begin{aligned}
& \Pi(0.5 i, 1)=\frac{1-i}{\sqrt{2}} \frac{\sqrt{\pi}}{2} \approx(0.7071+0.7071 i) \frac{\sqrt{\pi}}{2} \\
& \Pi(-0.5 i, 1)=\frac{1-i}{\sqrt{2}} \frac{\sqrt{\pi}}{2} \approx(0.7071-0.7071 i) \frac{\sqrt{\pi}}{2}
\end{aligned}
$$

\section{Multifactorials and fractional factorials}

Let $c_{n}$ be a sequence defined by Eqn. (3).

$$
c_{n}=(c * 1),(c * 2),(c * 3), \ldots,(c * n),
$$

where $c \neq 0$ is a constant multiplier of the terms of the sequence, called here as the factorial constant, and * represents multiplication. The product of the terms of the sequence may be called factorial of the sequence and represented as $\Pi(c, n)$. It is given as,

$$
\Pi(c, n)=(c)^{n} n !=c(c 2)(c 3) \ldots(c n)
$$

If $c$ is a fraction, the product may be called fractional factorial. For example, if $c=0.5$,

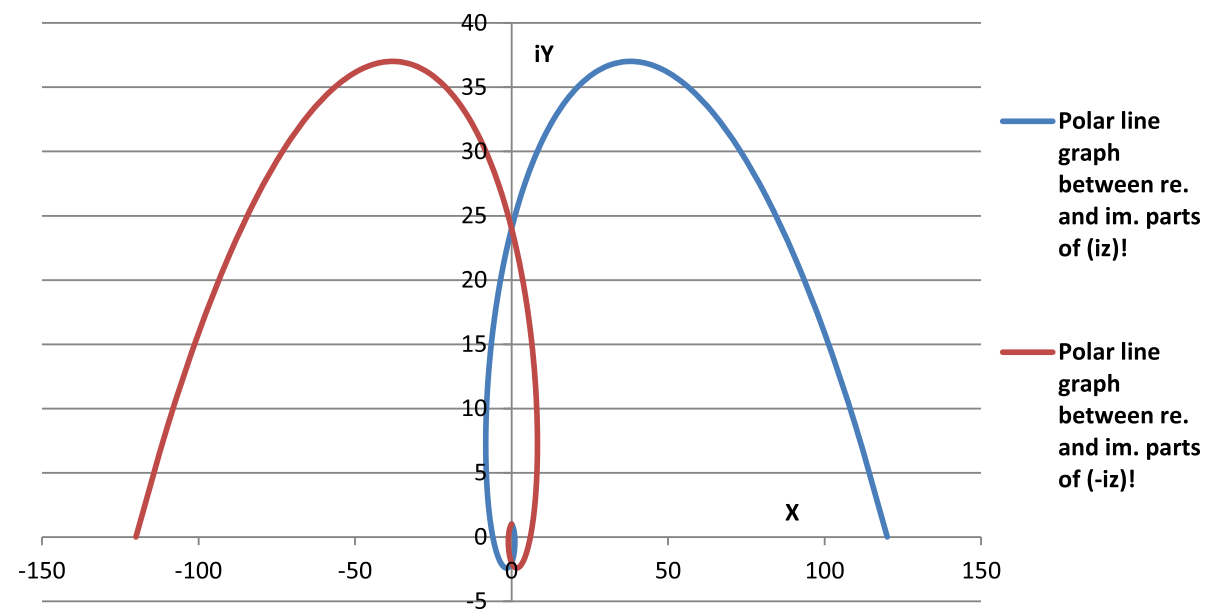

Figure 7 Polar line graph between real (X-axis) and imaginary (Y-axis) parts of complex factorials of imaginary numbers. 


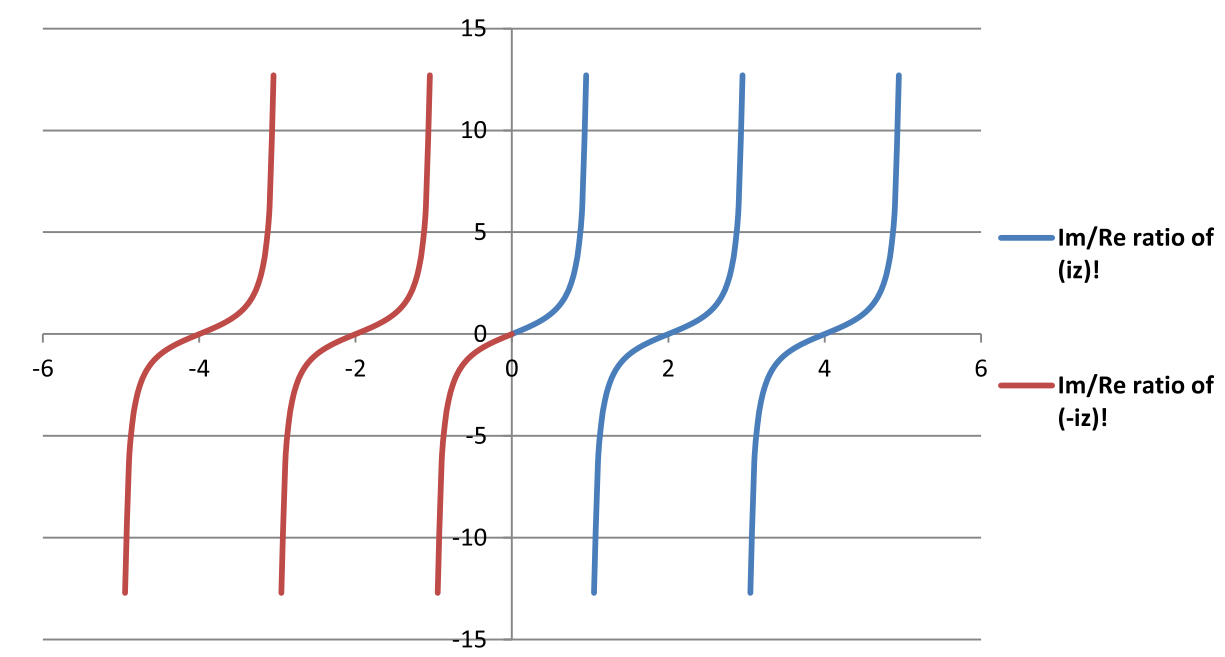

Figure 8 Ratio of imaginary to real parts of complex factorials of imaginary numbers (Y-axis) as per the present concept.

$$
\begin{aligned}
& \Pi(0.5, n)=(0.5)^{n} n !=(0.5 * 1)(0.5 * 2)(0.5 * 3) \ldots(0.5 * n) \\
& =(0.5)(1)(1.5) \ldots(0.5 * n)
\end{aligned}
$$

If $c$ is an integer $>1$, e.g., 2, 3 etc., we get multifactorials.

$$
\Pi(2, n)=(2)^{n} n !=(2 * 1)(2 * 2)(2 * 3) \ldots(2 n)=(2 n) ! !
$$

For example, if $c=2$, and $\mathrm{n}=4$, we get the double factorial of $c^{*} n=8$,

$$
\begin{aligned}
& \Pi(2,4)=(2)^{4} 4 !=(2 * 1)(2 * 2)(2 * 3)(2 * 4), \\
& =384=8 ! !
\end{aligned}
$$

Fractional factorials and multifactorials can be interpolated using Eqn. (5), where $c \neq 0$ is a fraction of a real or imaginary number.

$$
\left(c^{z}\right) z !=\Pi(c, z)=c^{z} \int_{0}^{\infty} t^{z} e^{-t} d t, z>0
$$

The value of fractional factorials and multifactorials at zero is 1. Fractional factorials and multifactorials of some real and imaginary numbers are given in Table 6 .

The modulus of the fractional factorials and multifactorials of real and imaginary numbers as proposed above follow recurrence relations:

$$
\Pi(c, z+1)=c(z+1) \Pi(c, z), c \neq 0
$$

\section{Table 5 Periodicity of factorials of imaginary numbers}

\begin{tabular}{llclc}
\hline $\mathbf{Z}$ & $(\mathbf{i z}) !$ & Coeff. & $(-\boldsymbol{i z}) !$ & Coeff. \\
\hline 1 & $\Pi(i, 1)=i \Pi(1)=i$ & $i$ & $\Pi(-i, 1)=-i \Pi(1)=-i$ & $-i$ \\
2 & $\Pi(i, 2)=-\Pi(2)=-2$ & -1 & $\Pi(-i, 2)=-\Pi(2)=-2$ & -1 \\
3 & $\Pi(i, 3)=-i \Pi(3)=-6 i$ & $-i$ & $\Pi(-i, 3)=i \Pi(3)=6 i$ & $i$ \\
4 & $\Pi(i, 4)=\Pi(4)=24$ & 1 & $\Pi(-i, 4)=\Pi(4)=24$ & 1 \\
\hline
\end{tabular}

\section{Gamma function}

As per the present concept, the Euler's gamma function (Eq. 2) may be modified as,

$$
\begin{aligned}
& \Gamma(c, z)=c^{z-1} \int_{0}^{\infty} t^{z-1} e^{-t} d t, z>0, c \neq 0 \\
& =\int_{0}^{\infty}(c t)^{z-1} e^{-t} d t, z>0, c \neq 0
\end{aligned}
$$

For real negative numbers the gamma will be,

$$
\begin{aligned}
& \Gamma(-1, z)=(-1)^{z-1} \int_{0}^{\infty} t^{z-1} e^{-t} d t, z>0 \\
& =\int_{0}^{\infty}(-t)^{z-1} e^{-t} d t, z>0
\end{aligned}
$$

For the negative $\mathrm{Z}$-axis, gamma will be given as,

$$
\Gamma(-1, z)=\int_{-\infty}^{0} t^{z-1} e^{t} d t, z>0
$$

The recurrence relation of negative gamma is:

$$
\Gamma(-1, z+1)=(-z) \Gamma(-1, z), z>0
$$

Negative gamma will be related to negative factorial function as follows:

$$
\Gamma(-1, z+1)=\Pi(-1, z), z>0
$$

Gamma values of real negative numbers are given in Table 7. Figure 9 represents the gamma of factorials 
Table 6 Fractional factorials and multifactorials

\begin{tabular}{cccccc}
\hline \multicolumn{3}{c}{ Fractional factorials and } & \multicolumn{3}{c}{ multifactorials of real positive numbers } \\
\hline & \multicolumn{4}{c}{ Fractional factorials } & \multicolumn{2}{c}{ Multifactorials } \\
\hline$z$ & $z !$ & $(0.5 z) !$ & $(1.5 z) !$ & $(2 z) ! !$ & $(3 z) ! ! !$ \\
0 & 1 & 1 & 1 & 1 & 1 \\
0.5 & 0.886 & 0.626 & 1.085 & 1.253 & 1.534 \\
1 & 1 & 0.5 & 1.5 & 2 & 3 \\
1.5 & 1.329 & 0.469 & 2.442 & 3.759 & 6.907 \\
2 & 2 & 0.5 & 4.5 & 8 & 18 \\
2.5 & 3.323 & 0.587 & 9.158 & 18.799 & 51.805 \\
3 & 6 & 0.75 & 20.25 & 48 & 162 \\
\hline
\end{tabular}

Fractional factorials and multifactorials of real negative numbers

\begin{tabular}{cccccc}
\hline & & \multicolumn{2}{c}{ Fractional factorials } & \multicolumn{2}{c}{ Multifactorials } \\
\hline$z$ & $(-z) !$ & $(-0.5) z !$ & $(-1.5 z) !$ & $(-2 z) ! !$ & $(-3 z) ! ! !$ \\
0 & 1 & 1 & 1 & 1 & 1 \\
0.5 & $0.886 i$ & $0.626 i$ & 1.085 & $1.253 i$ & $1.534 i$ \\
1 & -1 & -0.5 & -1.5 & -2 & -3 \\
1.5 & $-0.139 i$ & $-0.469 i$ & -2.442 & $-3.759 i$ & $-6.907 i$ \\
2 & 2 & 0.5 & 4.5 & 8 & 18 \\
2.5 & $3.323 i$ & 0.587 & 9.157 & $18.799 i$ & $51.805 i$ \\
3 & -6 & -0.75 & -20.25 & -48 & -162 \\
\hline
\end{tabular}

Fractional factorials and multifactorials of imaginary positive numbers

\begin{tabular}{cccccc}
\hline & \multicolumn{3}{c}{ Fractional factorials } & \multicolumn{2}{c}{ Multifactorials } \\
\hline$z$ & $(i z !)$ & $(0.5 i z) !$ & $(1.5 i z) !$ & $(2 i z) ! !$ & $(3 i z) ! ! !$ \\
0 & 1 & 1 & 1 & 1 & 1 \\
0.5 & $0.626+$ & $0.443+$ & $0.767+$ & $0.886+$ & $1.085+$ \\
& $0.626 i$ & $0.443 i$ & $0.767 i$ & $0.886 i$ & $1.085 i$ \\
1 & $i$ & $0.5 i$ & $1.5 i$ & $2 i$ & $3 i$ \\
1.5 & $-0.939+$ & $-0.332+$ & $-1.726+$ & $-2.658+$ & $-4.862+$ \\
& $0.939 i$ & $0.332 i$ & $1.726 i$ & $2.658 i$ & $4.862 i$ \\
2 & -2 & -0.5 & -4.5 & -8 & -18 \\
2.5 & $-2.349-$ & $-0.415-$ & $-6.475-$ & $-13.29-$ & $36.6-36.6 i$ \\
& $2.349 i$ & $0.415 i$ & $6.475 i$ & $13.29 i$ & \\
3 & $6 i$ & $-0.75 i$ & $-20.25 i$ & $-48 i$ & $-162 i$ \\
\hline
\end{tabular}

Fractional factorials and multifactorials of imaginary negative numbers

\begin{tabular}{cccccc}
\hline & & \multicolumn{2}{c}{ Fractional factorials } & \multicolumn{2}{c}{ Multifactorials } \\
\hline$z$ & $(-i z) !$ & $(-0.5 i z) !$ & $(-1.5 i z) !$ & $(-2 i z) ! !$ & $(-3 i z) ! !$ \\
0 & 1 & 1 & 1 & 1 & 1 \\
0.5 & $0.626-0.626 i$ & $0.443-0.443 i$ & $0.767-0.767 i$ & $0.886-0.886 i$ & $1.085-1.085 i$ \\
1 & $-i$ & $-0.5 i$ & $-1.5 i$ & $-2 i$ & $-3 i$ \\
1.5 & $-0.939-$ & $-0.332-$ & $-1.726-$ & $-2.658-$ & $-4.862-$ \\
& $0.939 i$ & $0.332 i$ & $1.726 i$ & $2.658 i$ & $4.862 i$ \\
2 & -2 & -0.5 & -4.5 & -8 & -18 \\
2.5 & $-2.349+$ & $-0.415+$ & $-6.475+$ & $-13.29+$ & $-36.6+$ \\
& $2.349 i$ & $0.415 i$ & $6.475 i$ & $13.29 i$ & $36.6 i$ \\
3 & $6 i$ & $0.75 i$ & $20.25 i$ & $48 i$ & $162 i$ \\
\hline
\end{tabular}

Table 7 Complex gamma of real negative and imaginary numbers

\begin{tabular}{|c|c|c|c|c|}
\hline \multicolumn{5}{|c|}{ Complex gamma of $(-z)$} \\
\hline$z$ & Real & Imaginary & Modulus & $\operatorname{Im} / \operatorname{Re}$ \\
\hline 0.05 & -19.230 & $-3.045 i$ & 19.470 & 0.158 \\
\hline 0.25 & -2.563 & $-2.563 i$ & 3.625 & 1 \\
\hline 0.5 & 0 & $-1.772 i$ & 1.772 & Comp Inf \\
\hline 0.75 & 0.866 & $-0.866 i$ & 1.225 & -1 \\
\hline 1 & 1 & 0 & 1 & 0 \\
\hline \multicolumn{5}{|c|}{ Complex gamma of (iz) } \\
\hline$z$ & Real & Imaginary & Modulus & $\operatorname{Im} / \mathrm{Re}$ \\
\hline 0.05 & 1.527 & $-19.410 i$ & 19.470 & -12.706 \\
\hline 0.25 & 1.387 & $-3.349 i$ & 3.625 & -2.414 \\
\hline 0.5 & 1.253 & $-1.253 i$ & 1.772 & -1 \\
\hline 0.75 & 1.132 & $-0.468 i$ & 1.225 & -0.414 \\
\hline 1 & 1 & 0 & 1 & 0 \\
\hline \multicolumn{5}{|c|}{ Complex gamma of $(-i z)$} \\
\hline$z$ & Real & Imaginary & Modulus & $\mathrm{Im} / \mathrm{Re}$ \\
\hline 0.05 & 19.410 & $1.527 i$ & 19.470 & 12.706 \\
\hline 0.25 & 3.349 & $1.387 i$ & 3.625 & 2.414 \\
\hline 0.5 & 1.253 & $1.253 i$ & 1.772 & 1 \\
\hline 0.75 & 0.468 & $1.132 i$ & 1.225 & 0.414 \\
\hline 1 & 0 & i & 1 & Comp Inf \\
\hline
\end{tabular}

of real negative numbers as per the present concept (Eqn. 14).

Similar to the gamma of real negative numbers (Eqn. 14), gamma of imaginary positive numbers will be

$$
\begin{aligned}
& \Gamma(i, z)=(i)^{z-1} \int_{0}^{\infty} t^{z-1} e^{-t} d t, z>0, \\
& =\int_{0}^{\infty}(i t)^{z-1} e^{-t} d t, z>0 .
\end{aligned}
$$

For imaginary negative numbers, gamma will be

$$
\begin{aligned}
& \Gamma(-i, z)=(-i)^{z-1} \int_{0}^{\infty} t^{z-1} e^{-t} d t, z>0, \\
& =\int_{0}^{\infty}(-i t)^{z-1} e^{-t} d t, z>0 \\
& =\int_{-\infty}^{0}(i t)^{z-1} e^{t} d t, z>0
\end{aligned}
$$

Figures 10 and 11 represent the gamma for imaginary numbers. 


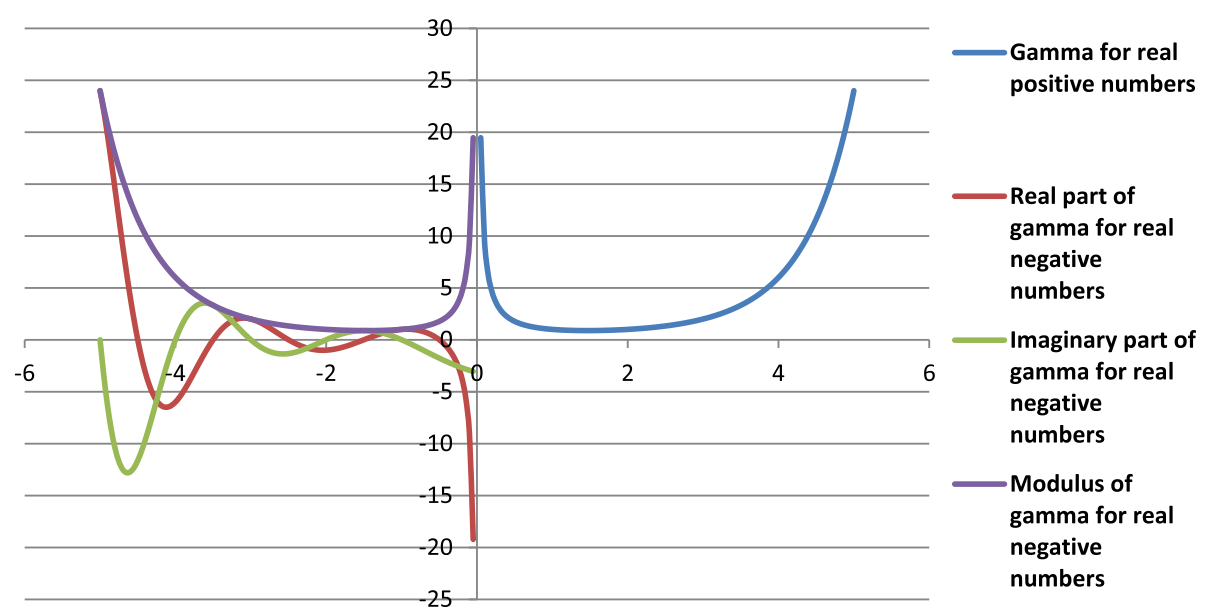

Figure $9 \mathrm{~A}$ plot of the gamma function for real numbers as per the proposed concept.

\section{Beta function}

The present concept of negative factorials can also be extended to beta function, also known as Euler's factorial of first kind. Beta may be defined as (Culham 2014; Weistein 2014c; Wikipedia 2014c):

$$
\begin{aligned}
& B(x, y)=\int_{0}^{1} \frac{t^{x-1}}{(1-t)^{y-1}} d t, x>0, y>0 \\
& =\frac{\Gamma(x) \Gamma(y)}{\Gamma(x+y)}, x>0, y>0 \\
& =\frac{(x-1) !(y-1) !}{(x+y-1) !}, x \geq 1, y \geq 1 .
\end{aligned}
$$

The gamma of negative numbers as per the present concept will be,

$$
\begin{aligned}
& \Gamma(-1, x)=(-1)^{x-1} \Gamma(1, x) ; x>0 \\
& \Gamma(-1, y)=(-1)^{y-1} \Gamma(1, y) ; y>0 \\
& \Gamma(-1, x+y)=(-1)^{x+y-1} \Gamma(1, x+y) ; x, y>0 .
\end{aligned}
$$

Therefore, the beta function of negative numbers, as per the present concept may therefore be defined as

$$
\begin{aligned}
& B(-x,-y)=\frac{(-1)^{x-1} \Gamma(1, x)(-1)^{y-1} \Gamma(1, y)}{(-1)^{x+y-1} \Gamma(1, x+y)}, x>0, y>0, \\
& =(-1)^{-1} B(x, y)=-B(x, y) ; x, y>0 .
\end{aligned}
$$

The graph of beta function is given in Figure 12.

It is seen from the historical account that the Euler's contributions to logarithms and gamma function have revolutionized developments in science and technology
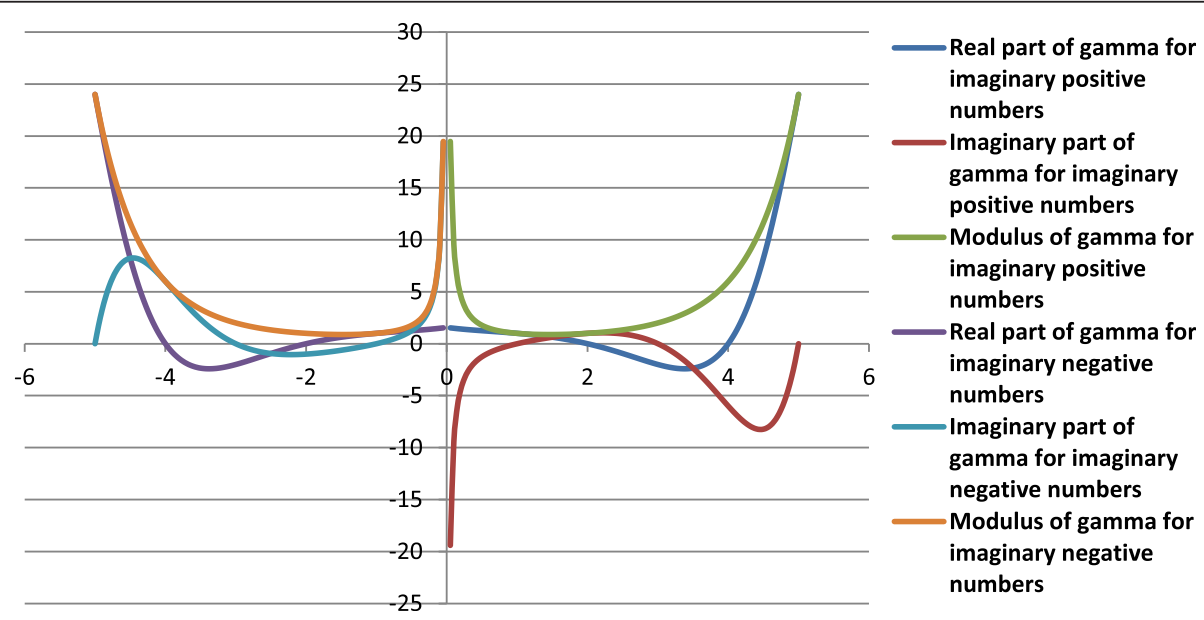

Figure $10 \mathrm{~A}$ plot of the gamma function for imaginary numbers as per the proposed concept. 

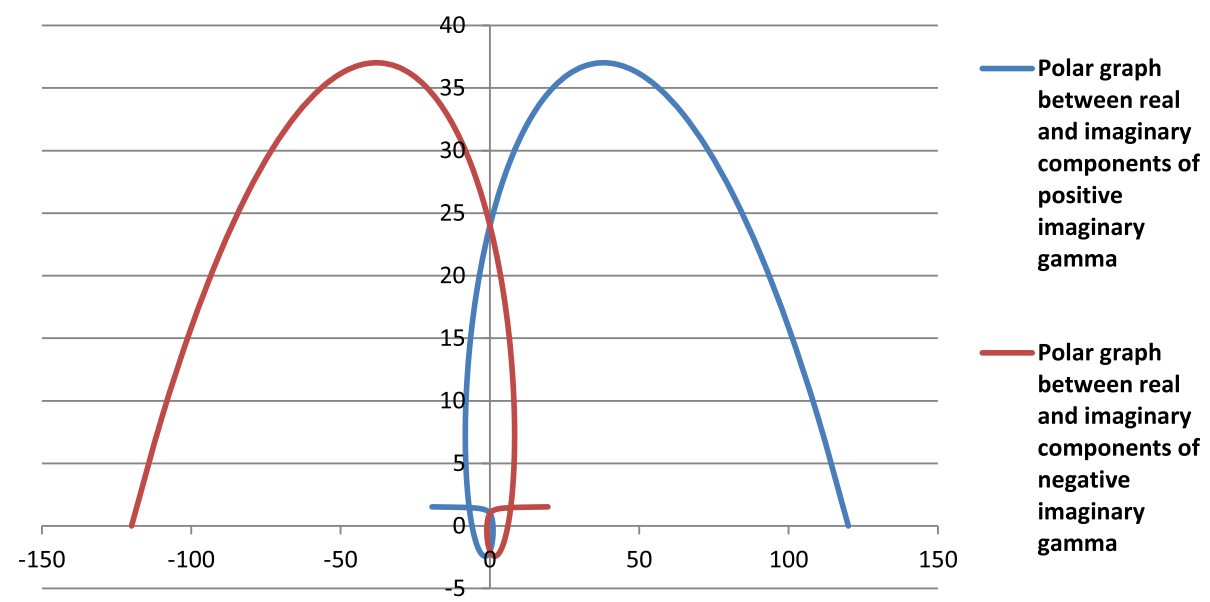

Figure 11 Polar graph for real (X-axis) vs. imaginary (Y-axis) components of gamma of imaginary numbers as per the present hypothesis.

(Lefort 2002; Lexa 2013). Factorial function was first defined for the positive real axis. Later its argument was shifted down by 1 , and the factorial function was extended to negative real axis and imaginary numbers. Recently, the author (Thukral and Parkash 2014; Thukral 2014) gave a new concept on the logarithms of real negative and imaginary numbers. Earlier the logarithms of real negative numbers were defined on the basis of hyperbola defined for the first quadrant and extended to the negative real axis, but the author defined the logarithms for the real negative axis on the basis of hyperbola located in the third quadrant. Similarly, the author in this paper has defined the factorial function for the real negative axis. The factorials of real and imaginary numbers thus defined show uniformity in magnitude and satisfy the basic factorial equation $(c)^{n} n !=c$ $(c 2)(c 3) \ldots(c n)$. Another lacuna in the existing Eularian concept of factorials is that although the factorials of negative integers are not defined, the double factorial of any negative odd integer may be defined, e.g., $(-1) ! !=1$, $(-3) ! !=-1,(-5) ! !=1 / 3$ etc. (Wikipedia 2014b). Another strange behaviour of double factorials is that as an empty product, $0 ! !=1$ but for non-negative even integer values, $0 ! !=\sqrt{\frac{2}{\pi}} \approx 0.7978$. The present concept will remove anomalies in factorials and double factorials. The present concept generalizes factorials as applicable to real and imaginary numbers, and fractional and mutifactorials.

\section{Conclusions}

The present paper examines the Eularian concept of factorials from basic principles and gives a new concept, based on the Eularian concept for factorials of real

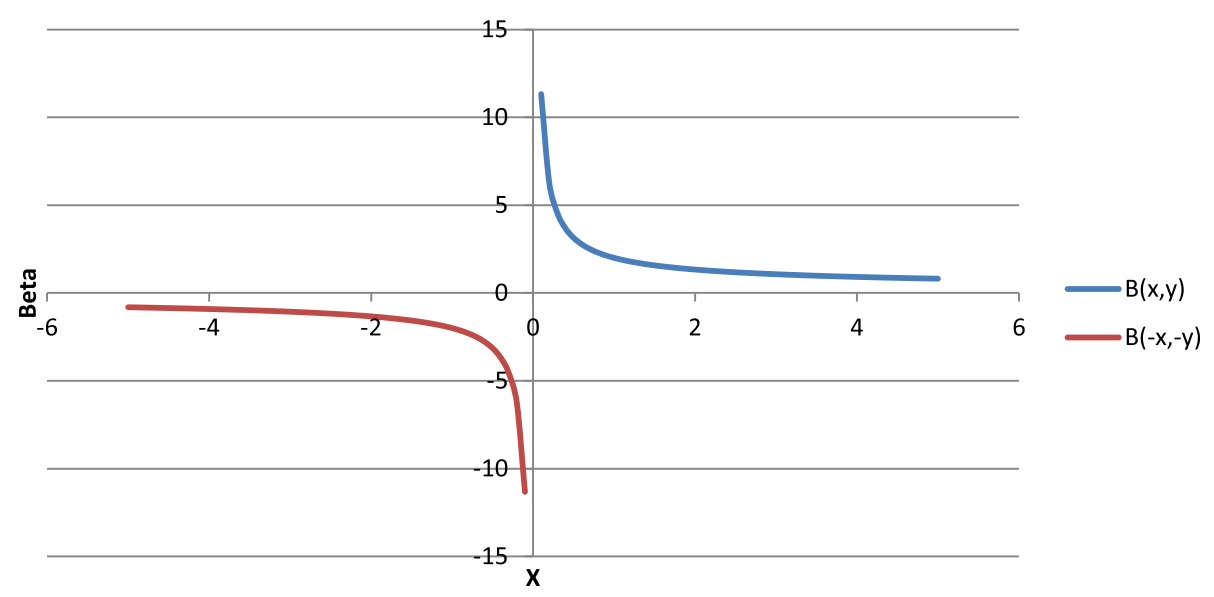

Figure 12 Graph for $\mathrm{B}(x, 0.5)$ and $\mathrm{B}(-x,-0.5)$ as per the present concept. 
negative and imaginary numbers. The factorials of positive and negative integers, and positive and negative imaginary number integers $(z)$, may be defined as $\Pi(c, z)=$ $c^{z} z !=c(c 2)(c 3) \ldots(c n)$, where $c$ is a constant $(+1,-1,+i$ or $-i$ ), and $z>0$. The factorials can be interpolated using

the Euler's modified integral equation, $\Pi(c, z)=\left(c^{z}\right) z$ ! $=c^{z} \int_{0}^{\infty} t^{z} e^{-t} d t, z>0$ for real and imaginary numbers. The factorials for real negative numbers may be defined by the integral equation, $\Pi(-1, z)=(-1)^{z} z !=\int_{-\infty}^{0} t^{z} e^{t} d t$, $z>0$. The factorials of negative real numbers are complex numbers. At negative integers the imaginary part of complex factorials is zero, and the factorials for $-1,-2$, $-3,-4$ are $-1,2,-6,24$ respectively. Similarly, the factorials of imaginary numbers are complex numbers. The moduli of negative real number factorials and imaginary number factorials are equal to the factorials of respective real positive numbers. The present paper also provides a general definition of fractional factorials and multifactorials. The factorials follow recurrence relations. Similarly, the Euler's gamma function has been redefined for negative real and imaginary numbers in a new perspective. Beta function on the real negative axis has also been redefined in the context of new concept. The present concept on factorials will be an improvement in the Euler's factorial and gamma functions.

\section{Software used}

Following softwares were used in this paper:

1. Wolfram Alpha Examples: Complex Numbers (http://www.wolframalpha.com/examples/ ComplexNumbers.html)

2. Draw Function Graphs - Recheronline. (http:// rechneronline.de/function-graphs/)

3. Definite integral calculator from Wolfram Alpha Widgets by Evan added in 2010 (http://www. wolframalpha.com/input/?i=definite\%20integral\% 20calculator)

4. Integral calculator from Wolfram Mathematica Online integrator (http://integrals.wolfram.com/index.jsp)

5. Gamma Function Evaluator - The Wolfram Functions site http://functions.wolfram.com/ webMathematica/FunctionEvaluation.jsp? name $=$ Gamma

6. Function calculator by XIAO gang 2012 (http://wims. unice.fr/wims/en_tool analysis function.en.html)

7. Microsoft Excel.

\section{Competing interests}

The author declares that he has no competing interests.

\section{Author's contributions}

The author conceived the idea, made the calculations and written and approved the manuscript.

\section{Acknowledgements}

Thanks are due to the Head, Department of Botanical \& Environmental Sciences, Guru Nanak Dev University, Amritsar, for research facilities.

Received: 8 October 2014 Accepted: 23 October 2014

Published: 6 November 2014

\section{References}

Anglani R, Barlie M (2007) Factorials as Sums. arXiv:Math/0702010v1 [Math.HO]. 1 Feb 2007. http://arxiv.org/pdf/math/0702010.pdf

Bhargava M (2000) The Factorial Function and Generalizations. Math. Assoc. America. Am. Math. Monthly. 107:783-799. http://www.jstor.org/stable/ 2695734? origin=JSTOR-pdf

Culham JR (2014) Factorial, Gamma and Beta Functions. Engineering Course ME755- Special Functions. University of Waterloo, Waterloo. http://mhtlab. uwaterloo.ca/courses/me755/web_chap1.pdf

Dartmouth College (2014) Correspondance Between Leonhard Euler and Chr. Goldbach, 1729-1763, pp 1-59. https://www.math.dartmouth.edu/ euler/ correspondence/fuss/goldbach1-59

Davis PJ (1959) Leonhard Euler's Integral: A Historical Profile of the Gamma Function. In Memorium: Milton Abramowitz. Math. Assoc. America. Amer Math Monthly. 66:849-869. http://www.jstor.org/stable/2309786? origin=JSTOR-pdf

Dutka J (1991) The early history of factorial function. Arch Hist Exact Sci 43(3):225-249

Gautschi W (2008) Leonhard Euler: His Life, the man, and his works. SIAM Rev 50(1):3-33. http://www.euler-2007.ch/doc/EulerLec.pdf

Gronau D (2003) Why is the gamma function so as it is? Teach Math Comput Sci 1:43-53. http://www.uni-graz.at/ gronau/TMCS_1_2003.pdf

Ibrahim AM (2013) Extension of factorial concept to negative numbers. Notes Theory Discrete Math 19:30-42. http://nntdm.net/papers/nntdm-19/NNTDM19-2-30_42.pdf

Lefort X (2002) History of the logarithms: an example of the development of a concept in mathematics. (In: Project Penelope, editor: Miguel Hernandez Gonzalez) Fundaciõn Canaria Orotava de Historia de la Ciencia, La Orotava. pp 142-155. http://fundacionorotava.org/archivos\%20adjuntos/ publicaciones/penelope/08_Lefort_Penelope.pdf

Lexa MA (2013) Remembering John Napier and his Logarithms. pp 1-13. www. see.ed.ac.uk/mlexa/supportingdocs/mlexa_napier_revised.pdf

Loeb DE (1995) A Generalization of the Binomial Coefficients. M.Sc. Report. Cornell University. pp 1-19. http://arxiv.org/abs/math/9502218v1

Luschny P (2014a) The Birth of the Real Factorial Function. pp 1-5. http://www. luschny.de/math/factorial/history.html

Luschny P (2014b) Is the Gamma Function mis-Defined? Or: Hadamard Versus Euler - Who Found the Better Gamma Function? p 31. http://www.luschny.de/ math/factorial/hadamard/HadamardsGammaFunctionMJ.html

Oldham KB, Myland J, Spanier J (2009) An Atlas of Functions: With Equator, the Atlas Function Calculator. Springer Science + Business Media, LLC, New York, p 750

Roman S (1992) The logarithmic binomial formula. Amer Math Month 99:641-648. http://www.jstor.org/stable/2324994

Srinivasan GK (2007) The gamma function: An eclectic tour. Amer Math Month 114:297-315. http://www.jstor.org/stable/pdfplus/27642193.pdf

Thukral AK (2014) Logarithms of imaginary numbers in rectangular form: A new technique. Can J Pure Appl Sc 8(3):3131-3137

Thukral AK, Parkash O (2014) A new approach for the logarithms of real negative numbers. Can J Pure Appl Sc 8(2):2955-2961.

Weistein EW (2014a) Factorial. MathWorld-A Wolfram Web Resource. pp 1-4. http://mathworld.wolfram.com/Factorial.html

Weistein EW (2014b) Double factorial. MathWorld-A Wolfram Web Resource. pp 1-3. http://mathworld.wolfram.com/DoubleFactorial.html

Weistein EW (2014c) Beta function. MathWorld-A Wolfram Web Resource. pp 1-3. http://mathworld.wolfram.com/BetaFunction.html 
Wikipedia (2014a) Factorial. pp 1-11. http://en.wikipedia.org/wiki/Factorial

Wikipedia (2014b) Double Factorial. pp 1-8. http://en.wikipedia.org/wiki/ Double_factorial

Wikipedia (2014c) Beta function. pp 1-5. http://en.wikipedia.org/wiki/ Beta_function

Wolfram Research (2014a) Factorial. pp 1-19. http://functions.wolfram.com/PDF/ Factorial.pdf

Wolfram Research (2014b) Gamma. pp 1-24. http://functions.wolfram.com/PDF/ Gamma.pdf

doi:10.1186/2193-1801-3-658

Cite this article as: Thukral: Factorials of real negative and imaginary

numbers - A new perspective. SpringerPlus 2014 3:658.

Submit your manuscript to a SpringerOpen ${ }^{\circ}$ journal and benefit from:

- Convenient online submission

- Rigorous peer review

- Immediate publication on acceptance

- Open access: articles freely available online

- High visibility within the field

- Retaining the copyright to your article

Submit your next manuscript at $\gg$ springeropen.com 